\title{
Factors Influencing Pain Expression in Patients with Cancer: An Expert Opinion
}

\author{
Sebastiano Mercadante (D) - Flaminia Coluzzi
}

Received: March 11, 2021 / Accepted: May 7, 2021 / Published online: May 20, 2021

(c) The Author(s) 2021

\begin{abstract}
Pain is a multidimensional experience that requires an appropriate assessment, and simple numbering may not be enough for the different components that are involved in the clinical expression. In consideration of the subjectivity of the symptom, each assessment should start from the way in which the patients perceive the pain and from how they deal with it. Some factors related to individual patient characteristics may make pain management difficult because of interference with the clinical pain expression. These factors may amplify the reporting of pain. Cognitive disorders and psychological distress seem to strongly influence pain expression and may render the analgesic treatment more difficult. Aberrant behaviors,
\end{abstract}

S. Mercadante $(\square)$

Anesthesia and Intensive Care Unit and Pain Relief and Supportive-Palliative Care Unit, La Maddalena Cancer Center, Via San Lorenzo 312, 90146

Palermo, Italy

e-mail: terapiadeldolore@lamaddalenanet.it;

03sebelle@gmail.com

F. Coluzzi

Department of Medical and Surgical Sciences and Biotechnologies, Sapienza University of Rome, Rome, Italy

F. Coluzzi

Sant'Andrea Hospital, Sapienza University of Rome, Rome, Italy such as alcoholism, smoking, and opioid misuse, may play a role, although geographic differences were found in terms of prevalence of the phenomenon, especially in some countries. Finally, the assessment of patients' expectation and the meaning of the personal feeling of changes in pain intensity provide new concepts in pain assessment, which may allow better personalization of the analgesic therapy. A modern pain assessment should include a multitude of factors influencing the phenotype of pain.

Keywords: Abuse; Alcoholism; Cancer pain; Opioids; Pain expression; Personal global impression; Psychological factor 


\section{Key Summary Points}

Some factors related to individual patient characteristics may make pain management difficult because of interference with the clinical pain expression.

Cognitive disorders and psychological distress may influence pain expression and may render the analgesic treatment more difficult.

Aberrant behaviors, such as alcoholism, smoking, and opioid misuse, may play a role.

Assessment of patients' expectation and the meaning of the personal feeling of changes in pain intensity may allow better personalization of the analgesic therapy.

\section{DIGITAL FEATURES}

This article is published with digital features, including a summary slide, to facilitate understanding of the article. To view digital features for this article go to https://doi.org/10.6084/ m9.figshare.14547081.

\section{INTRODUCTION}

Cancer pain is one of the most distressing symptoms in patients with cancer, particularly in the advanced stage of disease. The incidence of pain is variable ranging from $58 \%$ to $69 \%$ in patients with advanced cancer [1]. Pain is a multidimensional experience that requires an appropriate assessment for the different components that are involved in the clinical presentation. In consideration of the subjectivity of the symptom, assessment should start from the way in which the patient perceives the pain. Factors related to disease, pain mechanisms, or individual patient characteristics may make pain management difficult $[2,3]$. There are some other factors that may change pain expression, amplifying the reporting of pain [4-14]. Moreover, patients may express their personal goal of pain intensity, suggesting the need to individualize a specific treatment [15-18]. The aim of this review, based on expert opinion, is to assess the many confounding factors in pain assessment of patients with cancer, also considering some aspects aimed at individualizing the treatment.

Literature was selected for convenience, focusing on the most relevant and recent papers assessing these issues.

This article is based on previously conducted studies and does not contain any new studies with human participants or animals performed by any of the authors.

\section{MEASUREMENT OF PAIN}

Pain assessment is of paramount importance in patients with cancer, since it represents the basis for any analgesic intervention in daily practice. The pain measurement must be valid and reliable to allow a comparison at different intervals. The validity of a measurement refers to its appropriateness and usefulness, while reliability refers to the ability to deviate from a possible error, due to the interference of various factors, such as the patient's experience, operator, setting, or emotional factors.

Pain intensity can be evaluated by numerous methods. A one-dimensional scale of intensity or relief is commonly used. Multidimensional questionnaires include different aspects, not exclusively related to the intensity of pain, and are more commonly used for research. The most frequently used and validated scale is the numerical scale (NRS) (usually from 0 to 10) that has a common meaning even in different cultures. NRS is sensitive to changes in pain intensity after a therapeutic intervention [19]. To highlight a clinically appreciable variation, a 2-point decrease in the NRS or a percentage change of $33 \%$ has been reported to have good sensitivity and specificity [20].

On the basis of the interference of pain intensity with main daily functions, it was observed that a mild-bearable pain is between 0 
and 4. Beyond this level the pain interferes consistently with daily activities (moderate pain, between 5 and 6). Generally, this level of pain intensity indicates that a therapeutic adjustment is needed to achieve the goal of mild pain. Indeed, an intensity of pain between 7 and 10 (severe pain) represents a clinical imperative [21]. The relationship between these levels of range of pain intensity and some indicators of quality of life is not always linear, since no significant differences were found between absence of pain and mild pain, and between moderate pain and severe pain [22], and in some subjects the range of pain intensity can also be different, not always expressed in numbers. On the other hand, variations in NRS may still have a definite advantage for clinical decisions in most cases. NRS for pain intensity is one of the leading symptoms included in the Edmonton Symptom Assessment System (ESAS), which is the most common tool used to assess both physical and psychological symptoms by an unidimensional numeric rating scale that ranges from 0 (no symptom) to 10 (worse possible). ESAS is a self-reported, valid, and reliable tool for assessing the overall symptom burden, and it is sensitive to changes produced by a treatment [23].

\section{Pain Expression}

Many factors may confound and generally amplify the pain expression, i.e., the phenotype of pain. Pain is a subjective experience dependent on varying degrees by biological, psychological, and social factors. Thus, pain and nociception should be considered different phenomena, as the experience of pain does not correspond necessarily with the activity in sensory pathways.

\section{Cognitive Function}

Deterioration of cognitive function may induce a clinical misinterpretation, which may lead to inappropriate interventions. Cognitive dysfunction is often unrecognized or insufficiently diagnosed. As a consequence it is poorly managed by clinicians, even in hospital [4, 24]. Delirium may impair the assessment of physical symptoms, limiting the optimal symptom management [7]. Patients with cognitive dysfunction may report higher levels of pain. Delirium phenotype is mostly hypoactive, mixed, or hyperactive in a minority of cases. On the basis of a superficial recognition, physicians could be tempted to increase opioid doses, which may induce delirium, producing a selfmaintaining circuit. A retrospective study showed that patients who developed cognitive dysfunction during hospital admission expressed higher levels of symptom intensity, particularly pain [25]. In patients admitted to an acute palliative care unit, cognitive function improved after a comprehensive palliative care intervention. Indeed, non-responder patients reported an increase in symptom expression. Moreover, patients with normal cognitive function at admission, but whose condition deteriorated after admission, were unlikely to respond to treatments and had a short survival [4]. In studies performed in patients admitted to palliative care settings $[6,8,26]$, a large number of patients reported delirium. Despite a palliative care intervention, a large number of patients exhibited alterations in cognitive status after 1 week. An increase in intensity of a cluster of symptoms that are typically associated with a short survival was associated with the development of delirium. Indeed, patients who did not have delirium at admission and developed cognitive failure during admission were more likely to respond to a palliative intervention with an improvement of the cognitive status. Delirium rate has been reported to be different among palliative care settings. In an acute palliative care unit, $31.6 \%$ of patients had a diagnosis of delirium at admission. After a week of palliative care, the percentage dropped to $25.6 \%$. Depression and poor well-being were associated with delirium. The intensity of pain, depression, and global ESAS were significantly higher in patients with delirium. On the other hand, patients who developed delirium during admission displayed a higher level of symptom expression for pain, weakness, nausea, anxiety, dyspnea, and poor appetite. Of interest, in patients without delirium, there was an improvement of all ESAS items after 1 week of palliative care. Thus, symptom expression is 
amplified in patients with delirium, whereas patients without delirium can be more responsive to palliative treatments with a significant decrease in intensity of ESAS items [8].

In patients admitted to home care or hospices, generally with a short life expectancy, delirium could be due to a general derangement of the clinical condition commonly observed during the last weeks of life, rather than being attributable to reversible causes, e.g., opioids used for pain management $[27,28]$. As a matter of fact, although in home care or hospice settings the prevalence of delirium was similar to that reported in an acute setting, cognitive failure tended to worsen and responded poorly to a palliative care treatment [5].

Regardless of the poor possibilities to reverse delirium, cognitive function should be assessed even in medical environments at lower care intensity, e.g., home care or hospice. Of interest, delirium was missed by the referring team in about $60 \%$ of cases [4]. Thus, a continuous assessment of cognitive function is mandatory to detect the development of delirium. This approach could alert physicians to select patients with reversible causes of delirium, particularly in patients with a longer survival. Thus, screening for delirium is highly recommended in any palliative care settings, including hospice and home care, as well as in outpatient and inpatient oncology consultations.

\section{Psychological Distress}

Anxiety and depression are frequently reported in patients with advanced cancer and should be carefully considered during symptom assessment $[29,30]$. The expression of physical symptoms may be influenced among patients with advanced cancer experiencing anxiety and depression, because of somatization. Anxiety and depression were found to be associated with expression of physical symptoms [9]. In palliative care outpatients a significant association between pain and fatigue, quality of life, and mobility, and the presence of depression has been reported [31]. Pain intensity was independently associated with emotional distress. In a large sample of patients with advanced cancer, depression was independently associated with total ESAS. Indeed, the cross-sectional design does not allow one to draw conclusions regarding such a relationship [9]. In another study psychological distress was associated with higher pain intensity [32].

In a more recent study, the expression of most symptoms was affected by the presence of anxiety and depression. In particular, pain intensity was higher in patients with anxiety, whereas drowsiness and poor appetite more likely occurred in patients with depression. Of interest, these psychological symptoms were interdependent [33]. It has been reported that pain and anxiety may influence each other [34], and that depression may arise as a general common pathway in response to physical suffering and psychological distress [35]. A palliative care intervention was able to determine a decrease of ESAS symptoms intensity, but anxiety and depression continued to influence symptom hyperexpression, even at a lower level. This data suggests that patients experiencing anxiety and depression may have a less significant response to palliative care interventions [33].

Thus, patients with advanced cancer should undergo screening for depression and anxiety, as the psychological status may amplify pain expression and requires an individualized treatment.

\section{Aberrant Behaviors}

Alcoholism and Smoking Patients with addictive disorders, including alcoholism, have been often reported to be at greater risk for developing symptom hyperexpression and chemical coping [10]. The CAGE (Cut-down, Annoyed, Guilty, Eye-opener) questionnaire is a simple screening tool for alcoholism that is frequently used to detect alcoholism in studies of patients with advanced cancer [36]. CAGE correlated with specialistic interviews for detecting alcoholism [37]. The same questions in CAGE have been also used for detecting drug misuse (CAGE-AID) (see below).

CAGE-positive patients have been reported to be referred earlier to palliative care, also reporting higher symptom expression. Moreover, they were more frequently prescribed opioids. A comprehensive palliative care 
intervention has been reported to improve symptom intensity in both CAGE-positive and CAGE-negative patients with a minimal opioid dose escalation [12], confirming the data seen in a recent study [38]. In a study performed in Italy, however, there were no differences in symptom expression between CAGE-positive and CAGE-negative patients. This finding could be attributable to the low number of CAGE-positive patients who were selected from a large sample of patients. In Italy, CAGE-positive rate in patients with advanced cancer is low, ranging from $3.5 \%$ to $10 \%$ $[37,39,40]$, while in the USA, CAGE-positivity ranges from $17 \%$ to $28 \%$ of the cancer population $[11,12,36,41]$. The analysis of larger numbers of patients could provide more information about the role of alcoholism in symptom hyperexpression and the response to palliative care treatments.

Some factors, like tobacco use, alcohol, and illicit drug use, may be implicated in symptom expression, although their role remains controversial in the literature. In a retrospective study, smokers expressed higher pain intensity level and were more likely to be CAGE-positive [11]. Alcoholism has been reported to be associated with difficulties in controlling pain. Patients with a history of alcohol were more susceptible to developing addiction and to presenting poor compliance with the therapies, placing these patients at more risk of chemical coping. These patients are referred earlier to palliative care, have higher symptom expression, and are more frequently prescribed opioids [41]. Alcoholism has been associated with higher symptom distress, a history of smoking, and abuse or illicit use of drugs [41]. In a study in Italy, however, no differences in symptom expression were found, except for dyspnea, nausea, and well-being. Although higher doses of opioids were necessary for pain relief, the clinical response was globally not strongly influenced by alcoholism and smoking [39].

Thus, in patients with advanced cancer, smoking and alcoholism may have some implications in symptom expression and opioid response. However, data are controversial, as available data are not consistent to confirm these factors as relevant risk for symptom hyperexpression and opioid response.

Opioid Misuse In the last few years, there was a dramatic increase of opioid consumption in many countries, particularly in the USA. The misuse of opioid drugs in the USA and other developed countries represented a real emergency which resulted in an increase in the use of the health system and emergency visits $[42,43]$.

While the opioid crisis prevalently involved patients without cancer, there are many concerns about substance misuse and the possibility of diversion also in patients with cancer. The principal modalities of opioid-related aberrant behaviors are represented by addiction, dependence, chemical coping, and pseudo-addiction. The most common syndromes encountered in patients with cancer are chemical coping and pseudo-addiction [13, 14, 44]. While a diagnosis of chemical coping is made when a patient uses opioids to cope with stressful events, without a proper prescription, pseudo-addiction describes the phenomenon of patients with legitimate pain being undertreated, exhibiting drug-seeking behavior that is misinterpreted as addiction.

Surveys in patients of a palliative care program in the USA reported that about half of respondents, including patients and family members, were positive at screening urine tests [45-49].

The aberrant behaviors that may render the opioid treatment more complex are reported in Table $1[46,47]$.

From a retrospective analysis of data gathered in a pain clinic, high-risk patients tended to be younger, experienced more pain, had higher opioid requirements, and experienced more psychological symptoms [50, 51]. In another retrospective study of palliative care outpatients, about $20 \%$ of them were at risk of aberrant opioid behavior and 10\% were CAGEAID-positive. Aberrant opioid-use behaviors were associated with sex (men), pain, anxiety, and financial distress, and a positive CAGE-AID [49]. In a comparison study, patients at risk of aberrant opioid behavior were matched with patients with no risk. The presence of a clinical aberrant behavior was reported to be associated 
Table 1 Aberrant behaviors

Use of additional opioids other than those prescribed

Forget prescription

Sold prescription

Seeking euphoria from opioids

Admission to wanting opioids for anxiety, overdose, and death

Injected drug

Solicited opioids from other providers

Unauthorized emergency visits

Concurrent unauthorized dose escalation

Resisted therapy changes/alternative therapy

Reported lost or stolen prescriptions

Cancelled clinic visit

Requested early refills

Requested refills instead of clinic visit

Abused prescribed drug

with a younger age and higher levels of anxiety [45].

A diagnosis of chemical coping is made when a patient uses opioids to cope with stressful events, without a proper prescription. A distressing condition can induce a misuse of opioids. Chemical coping was diagnosed in about $18 \%$ of patients with cancer. Of interest, only $4 \%$ of patients had appropriate medical documentation. Chemical coping was associated with younger age, high pain intensity, poor well-being, CAGE positivity, and a better performance status [14]. Of interest, a comprehensive palliative care intervention may have an impact on the frequency of aberrant behavior and the use of opioid among patients with cancer on opioid therapy [48].

\section{PATIENT GOALS AND FEELING OF CHANGES IN PAIN INTENSITY}

Individuals learn the concept of pain and its treatment through their life experiences.
A person's report of an experience as pain should be accepted as such and properly considered during pain assessment. In other words, patients may have different feelings about the changes in pain intensity after a given treatment. For this reason treatments should be personalized according to patient goals and their capacity to perceive a clinical change after a specific analgesic treatment.

\section{Minimal Clinically Important Difference and Personalized Pain Intensity Goal}

ESAS has some limitations due to its subjectivity as individuals provide their own interpretation of symptom intensity with significant variations. Moreover, the clinical response to a pharmacological treatment is difficult to determine because of the lack of information about the minimal clinically important difference (MCID). MCID is the considered as the smallest amount of change needed to impact the patient's feeling of improvement or deterioration. Variable methods have been described to assess MCID. As there is a need to evaluate the individual variations in interpreting scales or numbers, the Patient's Global Impression (PGI) of change has been recently introduced and increasingly used. PGI is a validated tool to rate the subjective response based on the patients' feeling of improvement or deterioration, after receiving a specific treatment [52].

More recently, the personalized symptom goal (Patients' Pain Intensity Goal, PPIG) has been introduced. This is an assessment tool allowing one to tailor pain and symptom management, providing a personalized therapeutic "target" score $[15,16]$. The aim of a therapeutic strategy should be based on the achievement of the individual threshold for an intra-patient judgment. The Personalized Pain Goal Response (PPGR) is a meaningful concept related to PPIG, i.e, the achievement of the desired PPIG after a therapeutic intervention. The evaluation of PPIG and factors associated with PPGR and PGI would help physicians to personalize the pain management and to assess meaningful changes. This is fundamental in an acute setting where pain and symptom 
management are more intensive and rapid, because of expertise and frequent assessments, as timely therapeutic variations may provide adequate pain and symptom control in a short period.

In various studies most patients provided a PPIG of 3 or less $[15,16,18]$. While in some studies PPIG was unchanged at different intervals $[15,16,49]$, in another experience, PPIG decreased after 1 week, as patients would expect something more after achieving their initial target. The differences among these studies may be due to different time interval evaluations [18]. Thus, expectations can change according to a better clinical status.

Some studies reported that with a decrease of about 1 point after a comprehensive treatment is enough for patients to perceive a MCID $[17,49]$. However, the retrospective design, the longer and undetermined intervals among observations, the use of categories anchored to the well-being scale [53], or the setting, may have influenced the findings. In another study, patients perceived a MCID when the decrease in pain intensity was about 2 points. A judgment of better improvement and much better improvement was associated with a decrease in pain intensity of approximately 3.5 and 4.5 points, respectively. This observation is confirmed by pioneering studies which reported that patients perceive a meaningful clinical benefit with a reduction of at least 2 points in pain intensity or a $33 \%$ decrease of pain intensity from baseline [20].

Not all patients achieved their target expressed by PPIG. Of interest, PGI improvement was related to a higher baseline pain intensity. This finding could be attributed to a better feeling of improvement reported by patients when they experience a net decrease in pain intensity from baseline, e.g., halving the pain intensity, corresponding to about 2.5 points of difference. Similarly, higher PPIG was independently associated with a better PGI. This can be explained by the fact that the level of expectation is easier to be reached with a relatively small change in pain intensity. In other words, patients who reported a lower level of expectation (that is a high level of baseline pain intensity) seem to achieve better satisfaction. This observation was confirmed by a subsequent subanalysis of patients reporting higher values of PPIG. This group of patients required only minimal changes of pain intensity to achieve a good PGI [18]. In previous studies, the pain intensity recorded at initial assessment was considered as a negative factor for pain prognosis $[17,54-56]$. These studies showed undertreatment rather than an appropriate opioid dose titration. It is likely that this approach biased the outcome as a result of different factors. A very long time to stabilize the patients' condition, 8-22 days, was reported. This suggests non-standardized and non-optimized methods for pain management. In other studies, the retrospective design and long follow-up (3 weeks) in outpatients with only one therapeutic intervention possibly biased the outcome. Moreover, pain intensity should be considered as a dynamic concept that depends on when the patient is visited along the disease trajectory [57]. Pragmatic studies reported that adequate pain relief can be obtained within a few days using an adequate regimen of opioid dose titration in most patients [58-63]. Thus, PPIG, PPGR, and PGI should be taken into consideration for evaluating the effects of a comprehensive pain management, according to patients' expectations.

\section{CONCLUSION}

Pain is a multidimensional experience that requires an appropriate assessment. Simple numbering may not be enough for the different components that are involved in the clinical expression. In consideration of the subjectivity of pain phenotype, each assessment should start from the way in which the patient perceives the pain and from how they deal with it. Some factors related to specific patient characteristics may make pain management difficult because of interference with the clinical pain expression. These factors are able to amplify pain reporting and should be considered when planning a comprehensive management of pain. Cognitive dysfunction is often unrecognized or poorly diagnosed, and not managed by clinicians, even in hospital. Delirium may 
impair the recognition of physical symptoms, may induce misinterpretation of symptom assessment, and consequently may lead to inappropriate interventions. In patients with delirium, higher levels of pain may be the sign of hyperexpression and physicians may be mistakenly tempted to increase opioid doses, which are the possible cause of delirium, in a self-maintaining circuit. Screening for delirium in patients with advanced cancer with severe symptom intensity should be highly recommended in any setting where cancer pain is treated, including oncology inpatients and outpatient units, and the various palliative care settings, acute palliative care unit, hospices, and home care. Another aspect regards the psychological status of patients with cancer. Pain expression may vary in frequency and intensity among patients with advanced cancer and anxiety and depression because of somatization, i.e., the amplification of physical symptoms under psychological distress. Patients with addictive disorders, including alcoholism, are at greater risk for somatization and chemical coping. The association among some factors, like tobacco use, alcohol, and illicit drug use, implicated in symptom expression remains controversial in the literature. There are many concerns about substance misuse and the possibility of diversion in patients with cancer. After evaluation of the risk of opioid misuse, some clinical behaviors should be taken into consideration.

Finally, individuals learn the concept of pain and its treatment and may have different feelings about the changes in pain intensity after a given treatment. Thus, treatments should be personalized according to patient goals and the capacity to perceive a clinical change after a specific analgesic therapy is started. The personalized symptom goal is a new assessment tool to tailor pain and symptom management, providing a simple and individualized therapeutic "target" score. Therapeutic attempts should try to reach such a threshold for an intra-patient determination of a favorable response to a treatment.

Higher baseline intensity may paradoxically be associated with a better patient global impression of change, because they may feel an improvement with a net decrease in pain intensity. In other words, patients expecting a relatively high level of pain intensity are more likely to achieve better satisfaction. This recent finding contradicts previous studies in which the initial pain intensity had been considered a negative factor for pain prognosis, possibly because data were biased by undertreatment.

In conclusion, cognitive disorders and psychological distress seem to strongly influence pain expression and may render the pain management more difficult. Aberrant behaviors, such as alcoholism, smoking, and opioid misuse, may play a role, although geographic differences were found in terms of prevalence of these phenomena, especially in some countries. Finally, the assessment of patients' expectation and the meaning of the personal feeling of changes in pain intensity provide new concepts in pain assessment, which may allow better personalization of the analgesic therapy.

\section{ACKNOWLEDGEMENTS}

Funding. No funding or sponsorship was received for this study or publication of this article.

Authorship. All named authors meet the International Committee of Medical Journal Editors (ICMJE) criteria for authorship for this article, take responsibility for the integrity of the work as a whole, and have given their approval for this version to be published.

Authors' Contributions. Both authors contributed equally to finalize the paper.

Disclosures . Prof. Sebastiano Mercadante and Prof. Flaminia Coluzzi have nothing to disclose and no conflicts of interest to declare. Prof. Sebastiano Mercadante is a member of the journal's Editorial Board.

Compliance with Ethics Guidelines. This article is based on previously conducted studies and does not contain any new studies with 
human participants or animals performed by any of the authors.

Open Access. This article is licensed under a Creative Commons Attribution-NonCommercial 4.0 International License, which permits any non-commercial use, sharing, adaptation, distribution and reproduction in any medium or format, as long as you give appropriate credit to the original author(s) and the source, provide a link to the Creative Commons licence, and indicate if changes were made. The images or other third party material in this article are included in the article's Creative Commons licence, unless indicated otherwise in a credit line to the material. If material is not included in the article's Creative Commons licence and your intended use is not permitted by statutory regulation or exceeds the permitted use, you will need to obtain permission directly from the copyright holder. To view a copy of this licence, visit http://creativecommons.org/licenses/by$\mathrm{nc} / 4.0 /$.

\section{REFERENCES}

1. Bennett MI, Eisenberg E, Ahmedzai SH, et al. Standards for the management of cancer-related pain across Europe-a position paper from the EFIC Task Force on cancer pain. Eur J Pain. 2019;23:660-8.

2. Mercadante $S$. The patient with difficult cancer pain. Cancers (Basel). 2019;11(4):E565.

3. Portenoy RK, Ahmed E. Cancer pain syndromes. Hematol Oncol Clin North Am. 2018;32:371-86.

4. de la Cruz M, Fan J, Yennu S, et al. The frequency of missed delirium in patients referred to palliative care in a comprehensive cancer center. Support Care Cancer. 2015;23:2427-33.

5. Mercadante S, Masedu F, Balzani I, et al. Prevalence of delirium in advanced cancer patients in home care and hospice and outcomes after one week of palliative care. Support Care Cancer. 2018;26: 913-9.

6. Mercadante S, Adile C, Ferrera P, Cortegiani A, Casuccio A. Delirium assessed by memorial delirium assessment scale in advanced cancer patients admitted to an acute palliative/supportive care unit. Curr Med Res Opin. 2017;33:1303-8.
7. Delgado-Guay MO, Yennurajalingam S, Bruera E. Delirium with severe symptom expression related to hypercalcemia in a patient with advanced cancer: an interdisciplinary approach to treatment. J Pain Symptom Manag. 2008;36:442-9.

8. Mercadante S, Adile C, Ferrera P, Cortegiani A, Casuccio A. Symptom expression in advanced cancer patients admitted to an acute supportive/palliative care unit with and without delirium. Oncologist. 2019;24(6):e358-64.

9. Delgado-Guay M, Parsons HA, Li Z, et al. Symptom distress in advanced cancer patients with anxiety and depression in the palliative care setting. Support Care Cancer. 2009;17:573-9.

10. Tien AY, Schlaepfer TE, Fisch HU. Self-reported somatization symptoms associated with risk for extreme alcohol use. Arch Fam Med. 1998;1:33-7.

11. Kim YJ, Dev R, Reddy A, et al. Association between tobacco use, symptom expression, and alcohol and illicit drug use in advanced cancer patients. J Pain Symptom Manag. 2016;51:762-8.

12. Parson HA, Delgado-Guay MO, El Osta B, et al. Alcoholism screening in patients with advanced cancer: impact on symptom burden and opioid use. J Palliat Med. 2008;11:964-8.

13. Kwon JH, Tanco K, Hui D, Reddy A, Bruera E. Chemical coping versus pseudoaddiction in patients with cancer pain. Palliat Support Care. 2014;12:413-7.

14. Kwon JH, Tanco K, Park JC, et al. Frequency, predictors, and medical record documentation of chemical coping among advanced cancer patients. Oncologist. 2015;20:692-7.

15. Dalal S, Hui D, Nguyen L, et al. Achievement of personalized pain goal in cancer patients referred to a supportive care clinic at a comprehensive cancer center. Cancer. 2012;118:3869-77.

16. Hui D, Park M, Shamieh O, et al. Personalized symptom goals and response in patients with advanced cancer. Cancer. 2016;122:1774-81.

17. Arthur J, Tanco K, Park M, et al. Personalized pain goal as an outcome measure in routine cancer pain assessment. J Pain Symptom Manag. 2018;56:80-7.

18. Mercadante S, Adile C, Aielli F, et al. Personalized pain goals and responses in advanced cancer patients. Pain Med. 2020;21:e215-21.

19. Jensen MP. The validity and reliability of pain measures in adults with cancer. J Pain. 2003;4:2-21. 
20. Farrar JT, Portenoy RK, Berlin J, Kinman JL, Strom BL. Defining the clinically important difference in pain outcome measures. Pain. 2000;88:287-94.

21. Serlin RC, Mendoza TR, Nakamura Y, et al. When is cancer pain mild moderate or severe? Grading pain severity by its interference with function. Pain. 1995;61:277-84.

22. Wang X, Cleeland C, Mendoza T, et al. The effects of pain severity on health-related quality of life. Cancer. 1999;86:1845-55.

23. Hui D, Bruera E. The Edmonton symptom assessment system 25 years later: past, present, and future developments. J Pain Symptom Manag. 2017;53: 630-43.

24. Bruera E, Bush SH, Willey J, et al. Impact of delirium and recall on the level of distress in patients with advanced cancer and their family caregivers. Cancer. 2009;115:2004-12.

25. de la Cruz M, Yennu S, Liu D, Wu J, Reddy A, Bruera E. Increased symptom expression among patients with delirium admitted to an acute palliative care unit. J Palliat Med. 2017;20:638-41.

26. Mercadante S, Masedu F, Maltoni M, et al. Symptom expression in advanced cancer patients admitted to hospice or home care with and without delirium. Intern Emerg Med. 2019;14:515-20.

27. Mercadante S, Porzio G, Valle A, Aielli F, Casuccio A. Palliative sedation in patients with advanced cancer followed at home: a prospective study. J Pain Symptom Manag. 2014;47:860-6.

28. Mercadante S, Valle A, Porzio G, et al. How do cancer patients receiving palliative care at home die? A descriptive study. J Pain Symptom Manag. 2011;42:702-9.

29. Radbruch L, Nauck F, Ostgathe C, et al. What are the problems in palliative care? Results from a representative survey. Support Care. 2003;11:442-5.

30. Vignaroli E, Pace E, Willey J, et al. The Edmonton symptom assessment as a screening tool for depression and anxiety. J Palliat Med. 2006;9: 296-303.

31. Lloyd-Williams M, Shiels C, Taylor F, Dennis M. Depression-an independent predictor of early death in patients with advanced cancer. J Affect Disord. 2009;113:127-32.

32. Arthur J, Yennurajalingam S, Nguyen L, et al. The routine use of the Edmonton Classification System for Cancer Pain in an outpatient supportive care center. Palliat Support Care. 2015;13:1185-92.
33. Mercadante S, Adile C, Ferrera P, Cortegiani A, Casuccio A. Symptom hyper-expression in advanced cancer patients with anxiety and depression admitted to an acute supportive/palliative care unit. Support Care Cancer. 2019;27:3081-8.

34. Modonesi C, Scarpi E, Maltoni M, et al. Impact of palliative care unit admission on symptom control evaluated by the Edmonton Symptom Assessment System. J Pain Symptom Manag. 2005;30:367-73.

35. Lo C, Zimmermann C, Rydall A, et al. Longitudinal study of depressive symptoms in patients with metastatic gastrointestinal and lung cancer. J Clin Oncol. 2010;28:3084-9.

36. Dhalla S, Kopec JA. The CAGE questionnaire for alcohol misuse: a review of reliability and validity studies. Clin invest Med. 2007;1:33-41.

37. Mercadante S, Porzio G, Caruselli A, et al. The frequency of alcoholism in patients with advanced cancer admitted to an acute palliative care unit and a home care program. J Pain Symptom Manag. 2015;49:254-7.

38. Mercadante S, Adile C, Ferrera P, Casuccio A. Association between alcoholism and symptom expression, patient symptom goals, and clinical response in advanced cancer patients. Support Care Cancer. 2020;28:3361-9.

39. Mercadante S, Adile C, Ferrera P, Casuccio A. The effects of alcoholism and smoking on advanced cancer patients admitted to an acute supportive/palliative care unit. Support Care Cancer. 2017;25:2147-53.

40. Giusti R, Mazzotta M, Verna L, et al. The incidence of alcoholism in patients with advanced cancer receiving active treatment in two tertiary care centers in Italy. Alcohol Alcohol. 2019;54:47-50.

41. Bruera E, Moyano J, Seifert L, et al. The frequency of alcoholism among patients with pain due to terminal cancer. J Pain Symptom Manag. 1995;10: 599-603.

42. Passik SD, Kirsh KL, McDonald MV, et al. A pilot survey of aberrant drug-taking attitudes and behaviors in samples of cancer and AIDS patients. J Pain Symptom Manag. 2000;19:274-86.

43. Mercadante S. Potential strategies to combat the opioid crisis. Expert Opin Drug Saf. 2019;18: 211-21.

44. Kwon JH, Hui D, Bruera E. A pilot study to define chemical coping in cancer patients using the Delphi method. J Palliat Med. 2015;18:703-6. 
45. Arthur JA, Edwards T, Lu Z, et al. Frequency, predictors, and outcomes of urine drug testing among patients with advanced cancer on chronic opioid therapy at an outpatient supportive care clinic. Cancer. 2016;122:3732-9.

46. Arthur J, Bruera E. Balancing opioid analgesia with the risk of nonmedical opioid use in patients with cancer. Nat Rev Clin Oncol. 2019;16:213-26.

47. Arthur GA. Urine drug testing in cancer pain management. Oncologist. 2020;25:99-104.

48. Arthur J, Edwards T, Reddy S, et al. Outcomes of a specialized interdisciplinary approach for patients with cancer with aberrant opioid-related behavior. Oncologist. 2018;23:263-70.

49. Yennurajalingam S, Kang JH, Hui D, et al. Clinical response to an outpatient palliative care consultation in patients with advanced cancer and cancer pain. J Pain Symptom Manag. 2012;44:340-50.

50. Koyyalagunta D, Bruera E, Aigner C, Nusrat H, Driver L, Novy D. Risk stratification of opioid misuse among patients with cancer pain using the SOAPP-SF. Pain Med. 2013;14:667-75.

51. Carmichael AN, Morgan L, Del Fabbro E. Identifying and assessing the risk of opioid abuse in patients with cancer: an integrative review. Subst Abuse Rehabil. 2016;7:71-9.

52. Lauridsen $\mathrm{HH}$, Hartvigsen J, Manniche C, et al. Responsiveness and minimal clinically important difference for pain and disability instruments in low back pain patients. BMC Musculoskelet Disord. 2006;7:82.

53. Hui D, Bruera E. Minimal clinically important differences in the Edmonton Symptom Assessment System: the anchor is key. J Pain Symptom Manag. 2013;45:e4e5.

54. Apolone G, Corli O, Caraceni A, et al. Pattern and quality of care of cancer pain management. Results from the Cancer Pain Outcome Research Study Group. Br J Cancer. 2009;100:1566-7.

55. Fainsinger R, Fairchild A, Nekolaichuk C, Lawlor P, Lowe $\mathrm{S}$, Hanson $\mathrm{J}$. Is pain intensity a predictor of the complexity of cancer pain management? J Clin Oncol. 2009;7:585-90.

56. Knudsen AK, Brunelli C, Klepstad P, et al. Which domains should be included in a cancer pain classification system? Pain. 2012;153:696-703.

57. Mercadante S, Porzio G, Adile C, et al. Pain intensity as prognostic factor in cancer pain management. Pain Pract. 2015;15:E1-8.

58. Mercadante S, Gebbia V, David F, et al. Does pain intensity predict a poor opioid response in cancer patients? Eur J Cancer. 2011;47:713-7.

59. Mercadante S, Gebbia V, David F, et al. Tools for identifying cancer pain of predominantly neuropathic origin and opioid responsiveness in cancer patients. J Pain. 2009;10:594-600.

60. Mercadante S, Adile C, Caruselli A, et al. The palliative-supportive care unit in a comprehensive cancer center as crossroad for patients' oncological pathway. PLoS ONE. 2016;11(6):e0157300.

61. Hwang S, Chang V, Fairclough D, Kasimis B. Development of a cancer pain prognostic scale. J Pain Symptom Manag. 2002;24:366-78.

62. Kang JH, Kwon JH, Hui D, Yennurajalingam S, Bruera E. Changes in symptom intensity among cancer patients receiving outpatient palliative care. J Pain Symptom Manag. 2013;46:652-60.

63. Stromgren A, Groenvold M, Petersen M, et al. Pain characteristics and treatment outcome for advanced cancer patients during the first week of specialized palliative care. J Pain Symptom Manag. 2004;27:104-13. 